MSC 34G20

DOI: $10.14529 / \mathbf{m m p} 170301$

\title{
NORMAL FORMS OF THE DEGENERATE AUTONOMOUS DIFFERENTIAL EQUATIONS WITH THE MAXIMAL JORDAN CHAIN AND SIMPLE APPLICATIONS
}

\author{
L.R. Kim-Tyan ${ }^{1}$, B.V. Loginov ${ }^{2}$, Yu.B. Rousak ${ }^{3}$ \\ ${ }^{1}$ National University of Science and Technology "MISIS"(MISIS), Moscow, Russian \\ Federation, \\ ${ }^{2}$ Ulyanovsk State Technical University, Ulyanovsk, Russian Federation, \\ ${ }^{3}$ Department of Families, Housing, Community Services and Indigenous Affairs, \\ Canberra, Australia \\ E-mail: kim-tyan@yandex.ru, bvllbv@yandex.ru, irousak@gmail.com
}

\begin{abstract}
Degenerate differential equations, as part of the differential-algebraic equations, the last few decades cause increasing interest among researchers, both because of the attractiveness of the considered theoretical questions, and by virtue of their applications. Currently, advanced methods developed in this area are used for system modelling and analysis of electrical and electronic circuits, chemical reaction simulations, optimization theory and automatic control, and many other areas. In this paper, the theory of normal forms of differential equations, originated in the works of Poincare and recently developed in the works of Arnold and his school, adapted to the simplest case of a degenerate differential equations. For this purpose we are using technique of Jordan chains, which was widely used in various problems of bifurcation theory. We study the normal forms of degenerate differential equations in the case of the existence of the maximal Jordan chain. Two and three dimensional spaces are studied in detail. Normal forms are the simplest representatives of the degenerate differential equations, which are equivalent to more complex ones. Therefore, normal forms should be considered as a model type of degenerate differential equations.

Keywords: degenerate differential equations; normal forms; Jordan chains.
\end{abstract}

\section{Introduction}

The aim is to develop a technique of construction of normal forms for systems of differential equations with a degenerate operator at the derivative:

$$
A x^{\prime}=F(x, \mu), \quad F(0, \mu)=0 .
$$

Unless otherwise stated, it is assumed that $A, F: E_{1} \rightarrow E_{2}, \operatorname{dim} E_{1}=n, \operatorname{dim} E_{2}=n$, $(n=2,3$ and 4 ) where $A$ is a degenerate operator such as $\operatorname{dim} \operatorname{ker} A=1$ and $\operatorname{ker} A=\varphi$, ker $A^{*}=\psi$. Let function $F$ be sufficiently smooth and $B(\mu)$ be the linear part of the function $F$ calculated at the point $x=0$

$$
F(x, \mu)=B(\mu) x+R(x, \mu), \quad\|R(x, \mu)\|=o(\|x\|) .
$$

These results suggest the presence of Jordan chain consisting of $n$ elements for the operator-function $A-\varepsilon B_{0}\left(B_{0}=B(0)\right)$ and they are based on the symbiosis of ideas and 
approaches set forth in [1] and [2] for systems of ordinary differential equations without degeneration. Fundamentals of the theory of normal forms can be found in [3] and [4]. The work consists of two parts: the first part is algebraic in nature. Here the language of $A$-deformation of the operator $B_{0}=B(0)$ [2] and the apparatus of generalized Jordan chains (GJC) [1] are used to investigate the perturbations which do not change the Jordan structure of the operator-function. The second part of the work is presented in [5] and devoted to normal forms and bifurcations in the case of maximal Jordan chain, which consists of 2,3 or 4 elements. In constructing the normal forms for non-autonomous differential equations has been used the apparatus of the differential Jordan chains (DJC). Technically complicated case $n=4$ for non-autonomous differential equations which use the DJC and vector spaces over rings of polynomials (modules) is omitted, and published in the local press [6], and in [7]. It turned out that this type of the system describes aero elastic model for transonic circulating of gas flow around plates and envelopes [8].

\section{The Normal Form of the Non-Autonomous Degenerate Differential Equations, Depending on Parameters. Perturbations Which Keep the Jordan Structure of Equation}

Definition 1. The operator-function $B(\mu)$ is called an A-deformation of the operator $B_{0}=B(0)$ if for small $\mu$ the operator-function $A-\varepsilon B(\mu)$ has the same Jordan structure as the operator-function $A-\varepsilon B_{0}$.

Further in this article we consider that the operator-function $A-\varepsilon B_{0}$ has the maximal Jordan chain: $\varphi^{(1)}=\varphi, \varphi^{(2)}, \ldots, \varphi^{(p)}$, so $p=n$.

Lemma 1. If the operator-function $A-\varepsilon B_{0}$ has the maximal Jordan chain, then $B_{0}$ is an invertible operator.

Proof. The elements $\left\{\varphi^{(k)}\right\}_{1}^{p}$ of the Jordan chain of the operator-function $A-\varepsilon B_{0}$ are defined by relations

$$
A \varphi^{(k+1)}=B_{0} \varphi^{(k)}, \quad\left\langle B_{0} \varphi^{(k)}, \psi^{(1)}\right\rangle=0, \quad k=\overline{1, p-1} ; \quad\left\langle B_{0} \varphi^{(p)}, \psi\right\rangle \neq 0 .
$$

Because $p=n$ elements of the chain form a basis of $E_{1}$. If the operator $B_{0}$ is not invertible then exists an element $u$ of the space $E_{1}$ such as $B_{0} u=0$. Let $u=\xi_{1} \varphi^{(1)}+\ldots+\xi_{n-1} \varphi^{(n-1)}+\xi_{n} \varphi^{(n)}$ then $\xi_{1} B_{0} \varphi^{(1)}+\ldots+\xi_{n-1} B_{0} \varphi^{(n-1)}+\xi_{n} B_{0} \varphi^{(n)}=0$. From the condition $\left\langle B_{0} \varphi^{(k)}, \psi^{(1)}\right\rangle=\delta_{k n}$ follows that $\xi_{n}=0$. Then $\xi_{1} B_{0} \varphi^{(1)}+\ldots$ $+\xi_{n-1} B_{0} \varphi^{(n-1)}=A\left(\xi_{1} \varphi^{(2)}+\ldots+\xi_{n-1} \varphi^{(n)}\right)=0$ and therefore $\xi_{1} \varphi^{(2)}+\ldots+\xi_{n-1} \varphi^{(n)}=\lambda \varphi^{(1)}$ (because $\operatorname{ker} A=\varphi$ ). Since the elements $\varphi^{(1)}, \ldots, \varphi^{(n)}$ form the basis of the space, the $\xi_{1}=0, \quad \ldots, \quad \xi_{n-1}=0$ and $u=0$.

Corollary 1. If the $B(\mu)$ is A-deformation of $B_{0}$, then the equation (1) can be written as

$$
A(\mu) x^{\prime}=x+R_{1}(x, \mu),
$$

where the matrix of the operator $A(\mu)=B^{-1}(\mu) A$ has the form $[A(\mu)]=[C(\mu)] J[C(\mu)]^{-1}$. Here, the matrix $[C(\mu)]$ is formed by the columns of $\left[\varphi^{(1)}, \varphi^{(2)}(\mu), \ldots, \varphi^{(n)}(\mu)\right]$, and $J$ is Jordan block. 
Proof. Let us apply the matrix $[C(\mu)]^{-1}\left[B^{-1}(\mu) A\right][C(\mu)]$ to the vector $e_{i}=(0, \ldots, 1, \ldots, 0), \quad[C(\mu)]^{-1}\left[B^{-1}(\mu) A\right][C(\mu)] e_{i}=[C(\mu)]^{-1}\left[B^{-1}(\mu) A\right] \varphi^{(i)}(\mu)=$ $[C(\mu)]^{-1} \varphi^{(i-1)}(\mu)=e_{i-1}$, if $i>1$. When $i=1$, then $[C(\mu)]^{-1}\left[B^{-1}(\mu) A\right][C(\mu)] e_{1}=0$. Therefore $[C(\mu)]^{-1}\left[B^{-1}(\mu) A\right][C(\mu)]=J$.

Lemma 2. Let matrix $[C(\mu)]$ be a deformation of $E$ (identical matrix), where the first column has the form $[C(\mu)] e_{1}=a(\mu) \varphi, a(\mu) \neq 0, a(0)=1$. Then $[C(\mu)]$ defines the operator-function $B(\mu)$ - an A-deformation of the operator $B_{0}$ - up to the zero vector deformation $X(\mu), X(0)=0$.

Proof. Indeed, let the matrix $[C(\mu)]$ be such that $[C(0)]=\left[\varphi^{(1)}, \varphi^{(2)}, \ldots, \varphi^{(n)}\right]$. Then for sufficiently small $\mu$ matrix $[C(\mu)]$ is not degenerate, and vectors $\varphi^{(i)}(\mu)=[C(\mu)] e_{i}$ form a basis of the space $E_{1}$. We define a mapping $B(\mu)$ by formulas (using multiplier $1 / a(\mu)$ ): $B(\mu) \varphi^{(i)}(\mu)=A \varphi^{(i+1)}(\mu)$ when $i<n$, and $B(\mu) \varphi^{(n)}(\mu)=B(0) \varphi^{(p)}+X(\mu)$. Here $X(\mu)$ be an arbitrary deformation of vector 0 . The operator-function $A-\varepsilon B(\mu)$ has a maximal Jordan chain $\varphi^{(1)}, \varphi^{(2)}(\mu), \ldots, \varphi^{(n)}(\mu)$. Further, instead of operators we will consider their matrix in some basis $\left\{e_{1}, e_{2}, \ldots, e_{n}\right\}$.

Definition 2. The operator-function $B(\mu)$ is called A-versal deformation of the operator $B_{0}=B(0)$, if any $A$-deformation $\beta(\nu)$ of the operator $B_{0}$, can be obtained from $B(\mu)$ by replacing the parameter, i.e., there exists differentiable mapping $\mu=\kappa(\nu), \kappa(0)=0$, and the deformation $[S(\nu)]$ of the identical matrix $E$, such that

$$
\left[\beta^{-1}(\nu) A\right]=[S(\nu)]\left[B^{-1}(\kappa(\nu)) A\right][S(\nu)]^{-1} .
$$

Remark 1. It follows from (4) that the $\varphi^{(1)}$ is an eigenvector of the matrix $[S(\nu)]$. Indeed, $A \varphi^{(1)}=0 \Rightarrow A[S(\nu)]^{-1} \varphi^{(1)}=0 \Rightarrow[S(\nu)]^{-1} \varphi^{(1)}=\lambda \varphi^{(1)}$.

Lemma 3. Let $W$ be the subgroup of $\mathrm{GL}(n, R)$ of reversible matrix stretching vector $\varphi(\forall S \in W: S \varphi=\lambda \varphi, \lambda \neq 0)$ and $E_{\varphi}$ subspace $\operatorname{gl}(n, R)$ of all matrices for which $\varphi$ is an eigenvector. Through $\gamma_{W}(B)$ denote the orbit of matrix $B$ under the action of $W: \gamma_{W}(B)=\left\{S B S^{-1}, S \in W\right\}$. Then

$$
T_{B}\left(\gamma_{W}(B)\right)=\operatorname{Im}\left(L_{B} \mid E_{\varphi}\right)
$$

where $T_{B}\left(\gamma_{W}(B)\right)$ is the tangent space to the orbit $\gamma_{W}(B)$ at the point $B$ and $L_{B} \mid E_{\varphi}$ be the mapping defined by the formula $[\nu, B]$. Here $\nu \in E_{\varphi}, L_{B}=[\nu, B]$ is the commutator of $\nu$ and $B$ (Lie bracket).

Proof. If matrix $S$ is close to $E$ (identical) then $S$ belongs to $W$ if and only if $S=E+u$, where $u \in E_{\varphi}$ ( $u$ is small enough). Indeed if $S \in W$ then $(S-E) \varphi=(\lambda-1) \varphi$ and $u \in E_{\varphi}$,conversely if $u \in E_{\varphi}$ then $S \varphi=(1+\mu) \varphi, 1+\mu \neq 0$ because $S$ is invertible. Now $(E+\varepsilon u) B(E+\varepsilon u)^{-1}=(E+\varepsilon u) B(E-\varepsilon u+\ldots)=B+\varepsilon[u, B]+\ldots$, which proves $(5)$.

Remark 2. $\operatorname{codim}\left(\gamma_{W}(B)\right)=\operatorname{dim}\left(Z_{B} \cap E_{\varphi}\right)+\operatorname{codim}\left(E_{\varphi}\right)$. Here $Z_{B}=\operatorname{ker} L_{B}$ is the centralizer of the matrix $B$.

Indeed, $\operatorname{codim}\left(\gamma_{W}(B)\right)=\operatorname{dim} \operatorname{gl}(n, R)-\operatorname{dim}\left(\gamma_{W}(B)\right)=\operatorname{dim} \operatorname{gl}(n, R)-$ $-\operatorname{dim} \operatorname{Im}\left(L_{B} \mid E_{\varphi}\right)$, but $\operatorname{dim} \operatorname{Im}\left(L_{B} \mid E_{\varphi}\right)=\operatorname{dim} E_{\varphi}-\operatorname{dim} \operatorname{ker}\left(L_{B} \mid E_{\varphi}\right)=\operatorname{dim} E_{\varphi}-$ 
$-\operatorname{dim}\left(Z_{B} \cap E_{\varphi}\right)$. Consequently, $\operatorname{codim}\left(\gamma_{W}(B)\right)=\operatorname{dim} \operatorname{gl}(n, R)-\operatorname{dim} E_{\varphi}+$ $+\operatorname{dim}\left(Z_{B} \cap E_{\varphi}\right)=\operatorname{dim}\left(Z_{B} \cap E_{\varphi}\right)+\operatorname{codim}\left(E_{\varphi}\right)$.

Corollary 2. If $V$ is a manifold which belongs to $E_{\varphi}$, contains zero matrix $\mathbb{O}$ and intersects transversally $Z_{B} \cap E_{\varphi}$ at the point 0 , then $L_{B}\left(T_{0} V\right)=T_{B}\left(\gamma_{W}(B)\right)$. Here $T_{0} V$ is the tangent space to the manifold $V$ at the point 0 .

Proof. By the transversality, for each $\forall u \in E_{\varphi} \exists u_{1} \in T_{0} V$ and $u_{2} \in T_{0}\left(Z_{B} \cap E_{\varphi}\right)$ such that $u=u_{1}+u_{2} \Rightarrow L_{B}(u)=L_{B}\left(u_{1}+u_{2}\right)=L_{B}\left(u_{1}\right)$ and because of $u_{2} \in Z_{B}, \operatorname{Im}\left(L_{B} \mid T_{0} V\right)=$ $\operatorname{Im}\left(L_{B} \mid E_{\varphi}\right)=T_{B}\left(\gamma_{W}(B)\right)$.

Remark 3. If $B=J$, then $E_{\varphi}$ consists of matrices in which the first column has the form $(a, 0, \ldots, 0)$, and thus, $\operatorname{dim} E_{\varphi}=n^{2}-n+1$. On the other hand, it is known [2], that $Z_{J}$ is $T_{J}\left(\gamma_{W}(J)\right)$ span of matrices $E, J, J^{2}, \ldots, J^{n-1}$, each of which belongs $E_{\varphi}$ so $\operatorname{dim} T_{J}\left(\gamma_{W}(J)\right)=n^{2}-2 n+1$. So $V$ can be selected as subspace matrices

$$
\left(\begin{array}{cccc}
0 & 0 & \ldots & 0 \\
0 & v_{11} & \ldots & v_{1 n-1} \\
\ldots & \ldots & \ldots & \ldots \\
0 & v_{n-11} & \ldots & v_{n-1 n-1}
\end{array}\right) .
$$

Then the space $T_{J}\left(\gamma_{W}(J)\right)$ consists of matrices of the form $v J-J v$.

Indeed, by Corollary 2, $\operatorname{Im}\left(L_{J} \mid T_{0} V\right)=\operatorname{Im}\left(L_{J} \mid E_{\varphi}\right)=T_{J}\left(\gamma_{W}(J)\right)$, where $V$ is a submanifold of $E_{\varphi}$ contains zero matrix $\mathbb{O}$ and transversal to $Z_{J} \cap E_{\varphi}$ at zero.

Lemma 4. Let $B: \Lambda \rightarrow \operatorname{gl}(n, R) \in C^{1}$, where $\Lambda$ is neighbourhood of zero in $\mathbb{R}^{k}, B=$ $B(\lambda), B(0)=B_{0}$. We assume that $B(\lambda)$ transversal to $\gamma_{W}\left(B_{0}\right)$ at the point $\lambda=0$ and $k=\operatorname{codim}\left(\gamma_{W}\left(B_{0}\right)\right)$ and $V$ is a manifold which belongs to $E_{\varphi}$, contains zero matrix $\mathbb{O}$ and intersects transversally $Z_{B} \cap E_{\varphi}$ at the point 0 and such as $\operatorname{dim} V=\operatorname{dim} \gamma_{W}\left(B_{0}\right)$. Then the map $\Phi(v, \lambda): V \times \Lambda \rightarrow \operatorname{gl}(n, R)$, defined by the formula $\Phi(v, \lambda)=(E+v) B(\lambda)(E+v)^{-1}$, sets the local diffeomorphism of a neighborhood of the point $(0,0)$ in $V \times \Lambda$.

Proof. Let us calculate the derivative of $\Phi(v, \lambda)$ with respect to $v$ at the point $(0,0)$. It follows from the formula $(E+\varepsilon v) B(\lambda)(E+\varepsilon v)^{-1}=(E+\varepsilon v) B(\lambda)(E-\varepsilon v+\ldots)=$ $B(\lambda)+\varepsilon[v, B(\lambda)]+\ldots$ that derivative of $\Phi(v, \lambda)$ at the point $(0,0)$ is $[\cdot, B]$. According to Corollary 2 it maps the tangent space to the manifold $V$ to the tangent space to $\gamma_{W}\left(B_{0}\right)$. The derivative of $\Phi(v, \lambda)$ with respect to $\lambda$ at the point $(0,0)$ equals $B^{\prime}(0)-$ an arbitrary matrix from the tangent space $T B(0)$ (tangent space to $B(\lambda)$ at $\lambda=0$ ). Due to the manifold $V$ is transversal to $Z_{B} \cap E_{\varphi}$ at the point 0 the derivative of $\Phi(v, \lambda)$ at the point $(0,0)$ maps the tangent space to $V \times \Lambda$ to the whole space gl $(n, R)$. But, according to suppositions $k=\operatorname{codim}\left(\gamma_{W}\left(B_{0}\right)\right)$ and $\operatorname{dim} V=\operatorname{dim}\left(\gamma_{W}\left(B_{0}\right)\right)$, so $\operatorname{dim} V+\operatorname{dim} \Lambda=$ $\operatorname{dim} \mathrm{gl}(n, R)$ and therefore $\Phi(v, \lambda)$ is the local diffeomorphism.

Lemma 5. If $B=B_{0}^{-1} A$, and $U$ is subspace of $E_{\varphi}$ such that $E_{\varphi}=\left(Z_{B} \cap E_{\varphi}\right) \oplus U$, then the mapping $\Psi(u): U \rightarrow \mathrm{gl}(n, R)$, defined by the formula $\Psi(u)=(E+u) B(E+u)^{-1}$ defines a local diffeomorphism from a neighbourhood of zero in $U$ into the neighbourhood of $B$ in $\gamma_{W}(B)$. 
Proof. As in lemma 4 derivative of the mapping $\Psi(u)$ is equal to $[\cdot, B]$ and, by virtue of (5), it maps $U$ to $T_{B}\left(\gamma_{W}(B)\right)$. By construction, it has no zeros in $U$ and is therefore an isomorphism. Then $\Psi(u)$ is a local diffeomorphism.

Theorem 1. $B(\mu)$ is A-deformation of $B_{0}=B(0)$ ), if and only if $\left[B^{-1}(\mu) A\right]$ belongs to the submanifold $\gamma_{W}\left(B_{0}^{-1} A\right)$.

Proof. Necessity. By the Corollary 1, the matrix $\left[B^{-1}(\mu) A\right]$ is represented as $[C(\mu)] J[C(\mu)]^{-1}$ and respectively $\left[B^{-1}(0) A\right]=[C(0)] J[C(0)]^{-1}$, i.e. $\left[B^{-1}(\mu) A\right]=$ $[C(\mu)][C(0)]^{-1}\left[B_{0}^{-1} A\right][C(0)][C(\mu)]^{-1}$. In this case $[C(\mu)][C(0)]^{-1} \varphi=\lambda(\mu) \varphi, \lambda(\mu) \neq 0$, and consequently, $\left[B^{-1}(\mu) A\right]$ belongs to the submanifold $\gamma_{W}\left(B_{0}^{-1} A\right)$.

Sufficiency. If $\left[B^{-1}(\mu) A\right]$ belongs to the submanifold $\gamma_{W}\left(B_{0}^{-1} A\right)$, then for any $\mu\left[B^{-1}(\mu) A\right]=[C(\mu)]\left[B_{0}^{-1} A\right][C(\mu)]^{-1}=[C(\mu)][C(0)]^{-1}$. . $\left[B^{-1}(0) A\right][C(0)][C(\mu)]^{-1}=[S(\mu)] J[S(\mu)]^{-1}$, and by Lemma 2, $B(\mu)$ is $A$-deformation of $B_{0}$. Continuity (or differentiability if $B(\mu)$ is differentiable) of $C(\mu)$ follows from Lemma 5 . In this case $C(\mu)$ has the form $E+u(\mu)$, where $u(\mu)$ belongs to the subspace $U$, selected above.

Corollary 3. Let $B(\mu)$ be an A-deformation of $B_{0}=B(0)$. Then $B(\mu)$ is a versal A-deformation.

Proof. Indeed, let $\Delta(\nu)$ be other $A$-deformation of $B_{0}=\Delta(0)$ then $\left[\Delta^{-1}(\nu) A\right]=$ $[C(\nu)]\left[B_{0}^{-1} A\right][C(\nu)]^{-1}$. Introducing the function $\kappa(\nu)=\mu$, we obtain $\left[\Delta^{-1}(\nu) A\right]=$ $[C(\nu)]\left[B(\kappa(\nu))^{-1} A\right][C(\nu)]^{-1}$, where $\left[B(\kappa(\nu))^{-1} A\right]=B_{0}^{-1} A$.

Corollary 4. Let $B_{0}^{-1} A=J$, and $\mu=\left(\mu_{1}, \mu_{2}, \ldots, \mu_{k}\right)$ be a vector parameter, in order to $B(\mu)$ be a versal $A$-deformation of $B_{0}$, it is necessary to all $\partial B^{-1}(0) A / \partial \mu_{i}$ to have the following form. Let $U$ be arbitrary matrix (6) of size $(n-1)^{2}$, and $\check{U}_{i}$ be $n$-column $\left(u_{1 i}, \ldots, u_{n-1 i}, 0\right)$, and $\hat{U}_{i}$ be $n$-column $\left(0, u_{1 i}, \ldots, u_{n-1 i}\right)$. Then, according to Remark 3, matrix belonging to $T_{J}\left(\gamma_{W}(J)\right)$ must have the form $\left[0,-\check{U}_{1}, \hat{U}_{1}-\check{U}_{2}, \ldots, \hat{U}_{n-2}-\check{U}_{n-1}\right]$.

Remark 4. This condition is not sufficient.

Indeed, the matrix $U=\left(\begin{array}{ll}1 & 1 \\ 1 & 1\end{array}\right)$ generates a third-order matrix $\left(\begin{array}{ccc}0 & 1 & -1 \\ 0 & 1 & 0 \\ 0 & 0 & 1\end{array}\right)$ which could not be transformed to the single Jordan cell, because it has two different eigenvalues 0 and 1.

Theorem 2. Let the $B_{0}^{-1} A=J$ and $E_{i s}$ be the matrix in which 1 is in place $(i, s)$ and zeros are at the other places, and $s>i+1$. Then, for small $\varepsilon$ the matrix $J+\varepsilon E_{\text {is }}$ belongs to $\gamma_{W}(J)$ and $E_{i s}$ belongs to the tangent subspace $T_{J}\left(\gamma_{W}(J)\right)$.

Proof. Indeed, in the standard basis matrix $J+\varepsilon E_{i s}$ acts on it as follows: $0 \leftarrow e_{1} \leftarrow e_{2} \leftarrow$ $\cdots \leftarrow e_{s-1} ; e_{s-1}+\varepsilon e_{i} \leftarrow e_{s} ; \ldots ; e_{n-1} \leftarrow e_{n}$, in the basis $\varphi_{1}=e_{1} ; \ldots ; \varphi_{s-1}=e_{s-1} ; \varphi_{s}=$ $e_{s}-\varepsilon e_{i+1} ; \ldots ; \varphi_{2 s-i-2}=e_{2 s-i-2}-\varepsilon e_{s-1} ; \varphi_{2 s-i-1}=e_{2 s-i-1}-\varepsilon e_{s}+\varepsilon^{2} e_{i+1} ; \ldots$ it turns into the Jordan block. 
Remark 5. Any matrix, which has form $\left(\begin{array}{ccccc}0 & 1 & * & \ldots & * \\ 0 & 0 & 1 & \ldots & * \\ \ldots & \ldots & \ldots & \ldots & \ldots \\ 0 & 0 & 0 & \ldots & 1 \\ 0 & 0 & 0 & \ldots & 0\end{array}\right)$

belongs to $\gamma_{W}(J)$.

Indeed, it has one eigenvalue equal to zero and one eigenvector $e_{1}$, therefore, it could be reduced to the Jordan block.

Definition 3. Let $\Delta(\nu)$ be A-deformation of $B_{0}=B(0)$. We call $\Delta(\nu)$, induced from $B(\mu)\left(B(\mu)\right.$ is A-deformation of $\left.\left.B_{0}=B(0)\right)\right)$, with the help of the map $\mu=\kappa(\nu)$, if $\left[\Delta^{-1}(\nu) A\right]=\left[B^{-1}(\kappa(\nu)) A\right]$.

Definition 4. Let $B(\mu)$ be A-deformation of $B_{0}=B(0)$. We call $B(\mu)$ generating if any other $A$-deformation of $B_{0}($ say $\Delta(\nu))$ induced from $B(\mu)$ by means of some map $\mu=\kappa(\nu)$.

Theorem 3. If the dimension of the tangent space to the orbit of $B^{-1}(\mu) A$ at the point $\mu=0$ is equal to the dimension of the subspace $U$ (introduced in Lemma 5) then the deformation $B_{0}^{-1} A$ is generating.

Proof. It follows from the Lemma 5 that the dimension of the tangent space to the orbit of $B^{-1}(0) A$ at the point $\mu=0$ is equal to dimension of the tangent space to the orbit $\gamma_{W}\left(B_{0}^{-1} A\right)$. Therefore the mapping $B^{-1}(\mu) A$ defines a local diffeomorphism from the neighbourhood of the point $\mu=0$ to the neighbourhood $B_{0}^{-1} A$ in orbit $\gamma_{W}\left(B_{0}^{-1} A\right)$. Let $\Gamma$ be the inverse map such that for small $\mu, \mu \equiv \Gamma\left(B^{-1}(\mu) A\right)$. Then, if $\Delta(\nu)$ is an arbitrary $A$-deformation of $B_{0}=B(0)$, we can construct the mapping $\mu=\kappa(\nu)$ as $\mu=\Gamma\left(\Delta^{-1}(\nu) A\right)$. Receives $\left[\Delta^{-1}(\nu) A\right]=\left[B^{-1}(\kappa(\nu)) A\right]$.

Remark 6. If the perturbation $B^{-1}(\mu) A$ of $B_{0}^{-1} A$ is known, then it is possible to build the corresponding perturbation $B(\mu)$ of the operator $B_{0}$ as follows.

Let $B^{-1}(\mu) A=B_{0}^{-1} A+S(\mu)$, or $A=B(\mu)\left(B_{0}^{-1} A+S(\mu)\right)$. Since the perturbation $B_{0}^{-1} A+S(\mu)$ corresponds to some $A$-deformation of $B_{0}$, there is the maximal Jordan chain $\varphi^{(1)}, \varphi^{(2)}(\mu), \ldots, \varphi^{(n)}(\mu)$, for which $\left(B_{0}^{-1} A+S(\mu)\right) \varphi^{(1)}=0, \varphi^{(i)}(\mu)=\left(B_{0}^{-1} A+S(\mu)\right) \varphi^{(i+1)}(\mu)$ $(1<i<n)$. Therefore, if $\gamma(\mu)$ is the functional, determined by the conditions $\left\langle\varphi^{(1)}, \gamma(\mu)\right\rangle=1,\left\langle\varphi^{(k)}(\mu), \gamma(\mu)\right\rangle=0, k=2, \ldots, n$, then the operator $D(\mu)=B_{0}^{-1} A+$ $S(\mu)+\langle\cdot, \gamma(\mu)\rangle \varphi^{(n)}(\mu)$ is reversible [1]. Operator $D(\mu)$ is called the Schmidt's regularization for operator $B^{-1}(\mu) A$. Then $A=B(\mu)\left[D(\mu)-\langle\cdot, \gamma(\mu)\rangle \varphi^{(n)}(\mu)\right]=B(\mu) D(\mu)-$ $\langle\cdot, \gamma(\mu)\rangle B(\mu) \varphi^{(n)}(\mu)$, and by Lemma $2 B(\mu)=A D^{-1}(\mu)+\left\langle D^{-1}(\mu) \cdot \gamma(\mu)\right\rangle\left(B_{0} \varphi^{(n)}(0)+\right.$ $X(\mu))$.

\section{Normal Forms and Bifurcations in the Case of the Maximal Jordan Chain of Small Length}

A. The Jordan Chain of Length Two. Since $\|R(x, \mu)\|=o(\|x\|)$, in the case of the maximum Jordan chain of length 2 , and also in the non-parametric case, the degenerate differential equation in the basis of $\varphi^{(1)}, \varphi^{(2)}(\mu)$ could be transformed to the system: $x_{2}^{\prime}=x_{1}+f\left(x_{1}, x_{2}, \mu\right), 0=x_{2}+g\left(x_{1}, x_{2}, \mu\right)$, using reduction of Lyapunov - Schmidt [1]. 
The functions $f\left(x_{1}, x_{2}, \mu\right)$ and $g\left(x_{1}, x_{2}, \mu\right)$ satisfy conditions: $f(0,0, \mu) \equiv 0, g(0,0, \mu) \equiv 0$ и $D f(0,0, \mu) \equiv 0, D g(0,0, \mu) \equiv 0$. Using the change of variables $y_{1}=x_{1}+f\left(x_{1}, x_{2}, \mu\right)$, $y_{2}=x_{2}$ this system could be simplified $y_{2}^{\prime}=y_{1}, y_{2}=h\left(y_{1}, y_{2}, \mu\right)$. If the function $h\left(y_{1}, y_{2}, \mu\right)$ is smooth (analytical) and does not dependent on $y_{1}$, then in a neighbourhood of $(0,0)$ $y_{1}=0, y_{2}=0$ will be the only solution to this system. In general, by the theorem of implicit function, $y_{2}=G\left(y_{1}, \mu\right), G(0, \mu)=0, G^{\prime}(0, \mu)=0$ and therefore the system takes the form

$$
y_{2}^{\prime}=y_{1}, \quad y_{2}=y_{1}^{2} h\left(y_{1}, \mu\right), \quad\left|h\left(y_{1}, \mu\right)\right|=o\left(\left|y_{1}\right|+|\mu|\right) .
$$

In the simplest case, $h\left(y_{1}, \mu\right)=y_{1}+\mu$ we obtain the normal form of "loss of uniqueness" bifurcation $y_{2}^{\prime}=y_{1}, y_{2}=y_{1}^{2}\left(y_{1}+\mu\right)$. When $\mu=0, y_{1}(t) \equiv 0, y_{2}(t) \equiv 0$ is the only solution for zero initial value. When $\mu \neq 0$ this system could be transformed in to differential equation $\left(3 y_{1}^{2}+2 \mu y_{1}\right) y_{1}^{\prime}=y_{1}$ or if $y_{1} \neq 0$, to $\left(3 y_{1}+2 \mu\right) y_{1}^{\prime}=1$. Substitution $z=3 y_{1}+2 \mu$, $z^{\prime}=3 y_{1}$ allows to simplify the last equation to the form $z z^{\prime}=3$, which has a smooth solution in a neighborhood of $t=0$ with initial value $z(0)=2 \mu$. Thus, the uniqueness is violated.

Theorem 4. In order to system (7) has at the point $\mu=0$ the bifurcation "loss of uniqueness" for solution which starts at the point $(0,0)$ it is necessary, and in case $\partial h(0,0) / \partial \mu \neq 0$, it is sufficient, that $h(0,0)=0$.

Proof. Necessity. Assume the contrary: $h(0,0) \neq 0$. Then in a neighbourhood of $(0,0)$ $h\left(y_{1}, \mu\right)=A+y_{1} h_{1}\left(y_{1}, \mu\right)+\mu h_{2}\left(y_{1}, \mu\right), A \neq 0$. Differentiating the equation $y_{2}=y_{1}^{2} h_{1}\left(y_{1}, \mu\right)$ with respect to $t$, followed by replacement of $y_{2}^{\prime}$ to $y_{1}$ leads to an equation in a neighbourhood of $y_{1}=0$ without degeneracy for small $\mu$ :

$$
y_{1}^{\prime}=\left[2 A+3 y_{1} h_{1}\left(y_{1}, \mu\right)+y_{1}^{2} h_{1}^{\prime}\left(y_{1}, \mu\right)+2 \mu h_{2}\left(y_{1}, \mu\right)+\mu y_{1} h_{2}^{\prime}\left(y_{1}, \mu\right)\right]^{-1} .
$$

Thus, $\mu=0$ is a bifurcation point, as in any small $\mu$ exists two small solutions of (7), starting from the point $(0,0)$.

Sufficiency. Let the conditions $h(0,0)=0, \partial h(0,0) / \partial \mu \neq 0$. Then $h\left(y_{1}, \mu\right)=$ $y_{1} h_{1}\left(y_{1}, \mu\right)+\mu h_{2}\left(y_{1}, \mu\right)$, where $h_{2}(0,0) \neq 0$, hence $y_{2}=y_{1}^{3} h_{1}\left(y_{1}, \mu\right)+\mu y_{1}^{2} h_{2}\left(y_{1}, \mu\right)$, and $y_{2}=y_{1}^{3} h\left(y_{1}, 0\right)=y_{1}^{3} h\left(y_{1}\right)$ for $\mu=0$. Therefore, the first equation of the system (7) takes the form: $\left[3 y_{1}^{2} h\left(y_{1}\right)+y_{1}^{3} h^{\prime}\left(y_{1}\right)\right] y_{1}^{\prime}=y_{1}$. This equation has only one solution with initial value $y_{1}(0)=0$, namely $y_{1}(t) \equiv 0$. If $\mu \neq 0$, then $y_{2}=A(\mu) y_{1}^{2}+y_{1}^{3} h_{3}\left(y_{1}, \mu\right), A(\mu) \neq 0$, for small $y_{1}$ and $\mu$. Substituting expression for $y_{2}$ in the first equation of the system (7) for $y_{1} \neq 0$ gives $\left[2 A(\mu) y_{1}+y_{1}^{2} h_{4}\left(y_{1}, \mu\right)\right] y_{1}^{\prime}=y_{1} \Rightarrow y_{1}^{\prime}=\left[2 A(\mu) y_{1}+y_{1}^{2} h_{4}\left(y_{1}, \mu\right)\right]^{-1}$. This equation has a nonzero solution with the initial condition $y_{1}(0)=0$. Thus, $\mu=0$ is a bifurcation point.

B. The Jordan Chain of Length Three. In the case of the Jordan chain of length three, just like in the case $\mathbf{A}$, the degenerate differential equation in the basis of $\varphi^{(1)}, \varphi^{(2)}(\mu), \varphi^{(3)}(\mu)$ could be transformed to the system:

$$
y_{2}^{\prime}=y_{1}, \quad y_{3}^{\prime}=y_{2}+y_{1}^{2} f_{2}\left(y_{1}, y_{2}, \mu\right), \quad y_{3}=f_{3}\left(y_{1}, y_{2}, \mu\right) .
$$

Function $f_{3}$ satisfies the following conditions $f_{3}(0,0, \mu) \equiv 0$ and $D f_{3}(0,0, \mu) \equiv 0$. 
Consider the particular case of the system (8)

$$
y_{2}^{\prime}=y_{1}, \quad y_{3}^{\prime}=y_{2}, \quad y_{3}=\mu y_{1}^{2}+y_{2}^{2} .
$$

Let show that $\mu=0$ is a bifurcation point "changing of the domain of solution". First consider the system (9) in a punctured neighbourhood of $\mu=0,(\mu \neq 0)$. This system takes the form:

$$
y_{2}^{\prime}=y_{1}, \quad 2 \mu y_{1} y_{1}^{\prime}+2 y_{2} y_{1}=y_{2},
$$

which is equivalent to the system (when $\left.y_{1} \neq 0\right)$

$$
y_{2}^{\prime}=y_{1}^{2}, \quad 2 \mu y_{1}^{\prime}=y_{2}-2 y_{2} y_{1},
$$

because [9] the solutions of both of these systems have the same orbits (when $y_{1} \neq 0$ and $y_{2} \neq 0$ ) as the solutions of the equation $\frac{d y_{2}}{d y_{1}}=\frac{y_{1}^{2}}{y_{2}-2 y_{2} y_{1}}$. The system (11) by the Existence and Uniqueness theorem has solution started from each point $\left(y_{1}^{0}, y_{2}^{0}\right)$, in some neighbourhood of $(0,0)$. To clarify the behavior of solutions in a neighbourhood of $y=0$, $\mu=0$ we repeat the process of blowing-up of singularity by transition to the new variables $\left(y_{1}, u\right)$ where $y_{2}=u y_{1}^{2}$. Then $y_{2}^{\prime}=u^{\prime} \cdot y_{1}^{2}+u \cdot 2 y_{1}(1 / 2 \mu)\left(y_{2}-2 y_{2} y_{1}\right)=u^{\prime} \cdot y_{1}^{2}+u \cdot y_{1}(1 / \mu)(u$. $\left.y_{1}^{2}-2 u \cdot y_{1}^{3}\right)$.

By substituting this into (11) we obtain:

$$
u^{\prime}=1-u \cdot(1 / \mu)\left(u \cdot y_{1}-2 u \cdot y_{1}^{2}\right), \quad 2 \mu y_{1}^{\prime}=u \cdot y_{1}^{2}-2 u \cdot y_{1}^{3} .
$$

After this substitution the singular point $(0,0)$ "blow up" in the line $(0, u)$. Therefore, it is necessary to investigate all the singular points of the system (12) on this line. However, the vector field defined by the right of (12), has no singular points on this line, because when $y_{1}=0$ it is equal to $(1,0)$. To study the solutions of the original system go back to the variables $y_{1}, y_{2}=u \cdot y_{1}^{2}$. When $\mu=0$ system (9) takes the form $y_{2}^{\prime}=y_{1}, y_{3}^{\prime}=y_{2}$, $y_{3}=y_{2}^{2} \Rightarrow y_{2}^{\prime}=y_{1}, 2 y_{1} y_{2}=y_{2}$. If $y_{2} \neq 0$ these systems have the solution $y_{1}=1 / 2$, $y_{2}=y_{2}^{0}+t / 2, y_{3}=\left(y_{2}^{0}+t / 2\right)^{2}$.

Thus, in the first case $(\mu \neq 0)$, the manifold of the initial values of the solutions were two-dimensional, and in the second case - one-dimensional.

Remark 7. If for degenerate differential equation, with the maximal Jordan chain consisting from three elements, the non-linear part does not depend on the variable $y_{1}$, then it has no solutions in some deleted neighbourhood of the point $y=0$.

Indeed, the system (8) then takes the form $y_{2}^{\prime}=y_{1}, y_{3}^{\prime}=y_{2}, y_{3}=f_{3}\left(y_{2}, \mu\right) \Rightarrow y_{2}^{\prime}=y_{1}$, $f_{3}^{\prime}\left(y_{2}, \mu\right) y_{1}=y_{2}$ and since $D f_{3}(0, \mu) \equiv 0$, the second equation of the last system could be reduced by $y_{2}$ when $y_{2} \neq 0$ which gives $f_{4}\left(y_{2}, \mu\right) y_{1}=1$. It is obvious that this equation could not have small solutions, so $y \equiv 0$ is the only small solution of the system.

Remark 8. The last result does not take place for the degenerate equation with the maximal Jordan chain consisting of four elements. This is evident from example:

$$
y_{2}^{\prime}=y_{1}, \quad y_{3}^{\prime}=y_{2}, \quad y_{4}^{\prime}=y_{3}, \quad y_{4}=y_{2} \cdot y_{3} \Rightarrow y_{2}^{\prime}=y_{1}, \quad y_{3}^{\prime}=y_{2}, \quad y_{1} \cdot y_{3}+y_{2}^{2}=y_{3} .
$$

By substituting the second equation, we get: $y_{2}^{\prime}=y_{1}, 2 y_{1} y_{2}\left(1-y_{1}\right)^{-1}+y_{2}^{2}\left(1-y_{1}\right)^{-2} y_{1}^{\prime}=y_{2}$. 
If $y_{2} \neq 0$, the system takes form $y_{2}^{\prime}=y_{1}, y_{2} y_{1}^{\prime}=\left(1-y_{1}\right)^{2}-2 y_{1}\left(1-y_{1}\right)$. The system has a solution, starting at any point $\left(y_{1}^{0}, y_{2}^{0}\right), y_{2}^{0} \neq 0$.

Lemma 6. (A sufficient condition for the occurrence of bifurcation "of the changing of the domain of solution"). In order to point $\mu=0$ was a point of bifurcation "changes of the domain of solutions" for the system (8) in the neighbourhood of $y=0, \mu=0$, it is sufficient that the function $f_{3}\left(y_{1}, y_{2}, \mu\right)$ has the form $f_{3}\left(y_{1}, y_{2}, \mu\right)=h_{1}\left(y_{2}, \mu\right)+\mu h_{2}\left(y_{1}, y_{2}, \mu\right)$, where $\partial h_{1}(0, \mu) / \partial y_{2}=0, D h_{2}(0,0, \mu)=0$ and $D\left(\partial h_{2} / \partial y_{1}\right)(0,0, \mu) \neq 0$.

Indeed, if the $\mu=0$, then the system (8) takes the form $y_{2}^{\prime}=y_{1}, y_{3}^{\prime}=y_{2}+y_{1}^{2} f_{2}\left(y_{1}, y_{2}, 0\right)$, $y_{3}=h_{1}\left(y_{2}, 0\right)$. After substituting the third equation by the second: $y_{1} \partial h_{1}\left(y_{2}, \mu\right) / \partial y_{2}=y_{2}+$ $y_{1}^{2} f_{2}\left(y_{1}, y_{2}, 0\right)$, we can use the implicit function theorem in order to have $y_{2}=F\left(y_{1}\right)$. Thus, if $\mu=0$ solution of (8) lies on the curve $\left(y_{1}, F\left(y_{1}\right)\right)$, then the second equation of the system (8) can be written as $\left(\partial h_{1} / \partial y_{2}\right) y_{1}+\mu\left(\partial h_{2} / \partial y_{2}\right) y_{1}+\mu\left(\partial h_{2} / \partial y_{1}\right) y_{1}^{\prime}=y_{2}+y_{1}^{2} f_{2}\left(y_{1}, y_{2}, \mu\right)$. The system $\mu\left(\partial h_{2} / \partial y_{1}\right) y_{1}^{\prime}=y_{2}+y_{1}^{2} f_{2}\left(y_{1}, y_{2}, \mu\right)-\left(\partial h_{1} / \partial y_{2}\right) y_{1}-\mu\left(\partial h_{2} / \partial y_{2}\right) y_{1}, y_{2}^{\prime}=y_{1}$ when $\mu \neq 0$ has a solution in a neighbourhood of $(0,0)$ starting at any point $\left(y_{1}^{0}, y_{2}^{0}\right)$, to which $\left(\partial h_{2} / \partial y_{1}\right)\left(y_{1}^{0}, y_{2}^{0}, \mu\right) \neq 0$. Since $\left(\partial h_{2} / \partial y_{1}\right)(0,0, \mu)=0$ and $D\left(\partial h_{2} / \partial y_{1}\right)(0,0, \mu) \neq 0$, then by the implicit function theorem, the set of solutions of the equation $\left(\partial h_{2} / \partial y_{1}\right)\left(y_{1}^{0}, y_{2}^{0}, \mu\right)=0$ is one-dimensional, so there is a bifurcation "of the changing of the domain of solution".

Acknowledgements. This work was financially supported by the Ministry of Education and Science of Russia within the framework of stake tasks 2014/232. The study was conducted with financial support of RFBR, research project No 15-01-08599, 15-4102455p_povolgie_a.

\section{References}

1. Vainberg M.M., Trenogin V.A. Theory of Branching of Solutions of Non-Linear Equations. Leyden, Nordhoof International Publishing, 1974.

2. Arnold V.I. Geometricheskie metody v teoriy obyknovennykh differentsialnykh uravneniy [Geometrical Methods in the Theory of Ordinary Differential Equations]. Moscow, Moscow Center for Continuous Mathematical Education, 1999.

3. Shui-Nee Chow, Chengzhi Li, Duo Wang. Normal Forms and Bifurcation of Planar Vector Fields. Cambridge University Press, 1994.

4. Jooss G., Adelmeyer M. Topics in Bifurcation Theory and Applications. Singapore, New Jersey, London, Hong Kong, World Scientific, 1992.

5. Loginov B.V., Rousak Yu.B., Kim-Tyan L.R. Normal Forms of the Degenerate Differential Autonomous and Non-Autonomous Equations with the Maximal Jordan Chain of Length Two and Three. The Bulletin of Irkutsk State University. Series: Mathematics, 2015, vol. 12, pp. 58-71. (in Russian)

6. Loginov B.V., Rousak Yu.B., Kim-Tyan L.R. Normal Forms for the Degenerate NonAutonomous Differential Equations in the Spaces $R^{n}, n=2,3,4$. Sbornik nauchnykh trudov "Prikladnaya matematika i mekhanika", Ulyanovsk, 2014, no. 10, pp. 142-160. (in Russian)

7. Loginov B.V., Rousak Yu.B., Kim-Tyan L.R. Differential Equations with Degenerated Variable Operator at the Derivative. Current Trends in Analysis and Its Applications. Proceedings of the 9th ISAAC Congress, Krakow 2013, 2015, pp. 101-108. DOI: 10.1007/9783-319-12577-0_14 
8. Marszalek W. Fold Points and Singularity Induced Bifurcation in Inviscid Transonic Flow. Physics Letters A, 2012, vol. 376, issues 28-29, pp. 2032-2037. DOI:10.1016/j.physleta.2012.05.003

9. Stepanov V.V. Kurs differentsialnykh uravneniy [The Course of the Differential Equations]. Moscow, Gosudarstvennoe izdatel'stvo tekhniko-teoreticheskoy literatury, 1950.

Received April 8, 2015

\title{
НОРМАЛЬНЫЕ ФОРМЫ ВЫРОЖДДННЫХ АВТОНОМНЫХ ДИФФЕРЕНЦИАЛЬНЫХ УРАВНЕНИЙ С МАКСИМАЛЬНОЙ ЖОРДАНОВОЙ ЦЕПОЧКОЙ И ПРОСТЕЙШИЕ ПРИЛОЖЕНИЯ
}

\author{
Л.Р. Ким-Тян ${ }^{1}$, Б.В. Логинов ${ }^{2}$ Ю.Б. Русак ${ }^{3}$ \\ ${ }^{1}$ Национальный исследовательский технологический университет МИСиС, \\ г. Москва \\ 2 Ульяновский государственный технический университет, г. Ульяновск \\ 3 Департамент социального сервиса, г. Канберра, Австралия
}

\begin{abstract}
Вырожденные дифференциальные уравнения, как часть алгебродифференциальньх уравнений, последние десятилетия вызывают все больший интерес среди исследователей, как в силу привлекательности рассматриваемых теоретических вопросов, так и в силу их приложений. В настоящее время развитые в данной области методы используются для системного моделирования и анализа электрических и электронных цепей, моделирования химических реакций, теории оптимизации и автоматического регулирования, а также во многих других областях. В настоящей работе теория нормальных форм дифференциальных уравнений, берущая свое начало в работах А. Пуанкаре, а последнее время развиваемая в работах В.И. Арнольда и его учеников, адаптируется к простейшим случаям вырожденных дифференциальных уравнений. Для этого существенно используется техника жордановых цепочек, давно и широко используемая в различных задачах теории бифуркации. Изучаются нормальные формы вырожденных дифференциальных уравнений в случае существования максимальной жордановой цепочки. Подробно изучаются случаи размерностей 2 и 3. Нормальные формы являются единственно возможными представителями вырожденных дифференциальных уравнений, сводящихся к своей нормальной форме. Поэтому нормальные формы следует считать модельными.

Ключевые слова: вырожденные дифференииальные уравнения; нормальные формы; жсордановы цепочки.
\end{abstract}

\section{Литература}

1. Вайнберг, М.М. Теория ветвления решений нелинейных уравнений / М.М. Вайнберг, В.А. Треногин. - М.: Наука, 1969.

2. Арнольд, В.И. Геометрические методы в теории обыкновенных дифференциальных уравнений / В.И. Арнольд. - М.: Московский центр непрерывного математического образования, 1999. 
3. Ван, Д. Нормальные формы и бифуркации векторных полей на плоскости / Д. Ван, Ч. Ли, Ш.-Н. Чоу. - М.: Московский центр непрерывного математического образования, 2005.

4. Jooss, G. Topics in Bifurcation Theory and Applications / G. Jooss, M. Adelmeyer. Singapore; New Jersey; London; Hong Kong: World Scientific, 1992.

5. Логинов, Б.В. Нормальные формы вырожденных автономных и неавтономных дифференциальных уравнений с максимальной жордановой цепочкой длины два и три / Б.В. Логинов, Ю.Б. Русак, Л.Р. Ким-Тян // Известия Иркутского государственного университета. Серия: Математика. - 2015. - Т. 12. - С. 58-71.

6. Логинов, Б.В. Нормальные формы для вырожденных неавтономных дифференциальных уравнений в пространствах $R^{n}, \mathrm{n}=2,3,4$ / Б.В. Логинов, Ю.Б. Русак, Л.Р. КимТян // Сборник научных трудов «Прикладная математика и механика». - Ульяновск: УлГТУ, 2014. - № 10. - С. 142-160.

7. Loginov, B.V. Differential Equations with Degenerated Variable Operator at the Derivative / B.V. Loginov, Yu.B. Rousak, L.R. Kim-Tyan // Current Trends in Analysis and Its Applications. Proceedings of the 9th ISAAC Congress. - 2015. - P. 101-108.

8. Marszalek, W. Fold Points and Singularity Induced Bifurcation in Inviscid Transonic Flow / W. Marszalek // Physics Letters A. - 2012. - V. 376, issues 28-29. - P. 2032-2037.

9. Степанов, В.В. Курс дифференциальных уравнений / В.В. Степанов. - М.: Гос. изд-во технико-теоретической литературы, 1950.

Луиза Ревмировна Ким-Тян, кандидат физико-математических наук, кафедра «Математика», Национальный исследовательский технологический университет МИСиС(г. Москва, Российская Федерация), kim-tyan@yandex.ru.

Борис Владимирович Логинов, доктор физико-математических наук, профессор, кафедра «Высшая математика», Ульяновский государственный технический университет (г. Ульяновск, Российская Федерация), bvllbv@yandex.ru.

Юрий Борисович Русак, кандидат физико-математических наук, доцент, Департамент социального сервиса (г. Канберра, Австралия), irousak@gmail.com.

Работа выполнена в рамках государственного задания № 2014/232 Минобрнауки России и при поддержке грантов РФФИ 15-01-08599, 15-41-02455p.

Поступила в редакиию 8 апреля 2015 г. 